Prepared for the U.S. Department of Energy under Contract DE-AC05-76RL01830

\title{
Analytical Data Report of Water Samples Collected From BP-5 Operable Unit A Well (C5858)
}

Michael Lindberg

September 2008

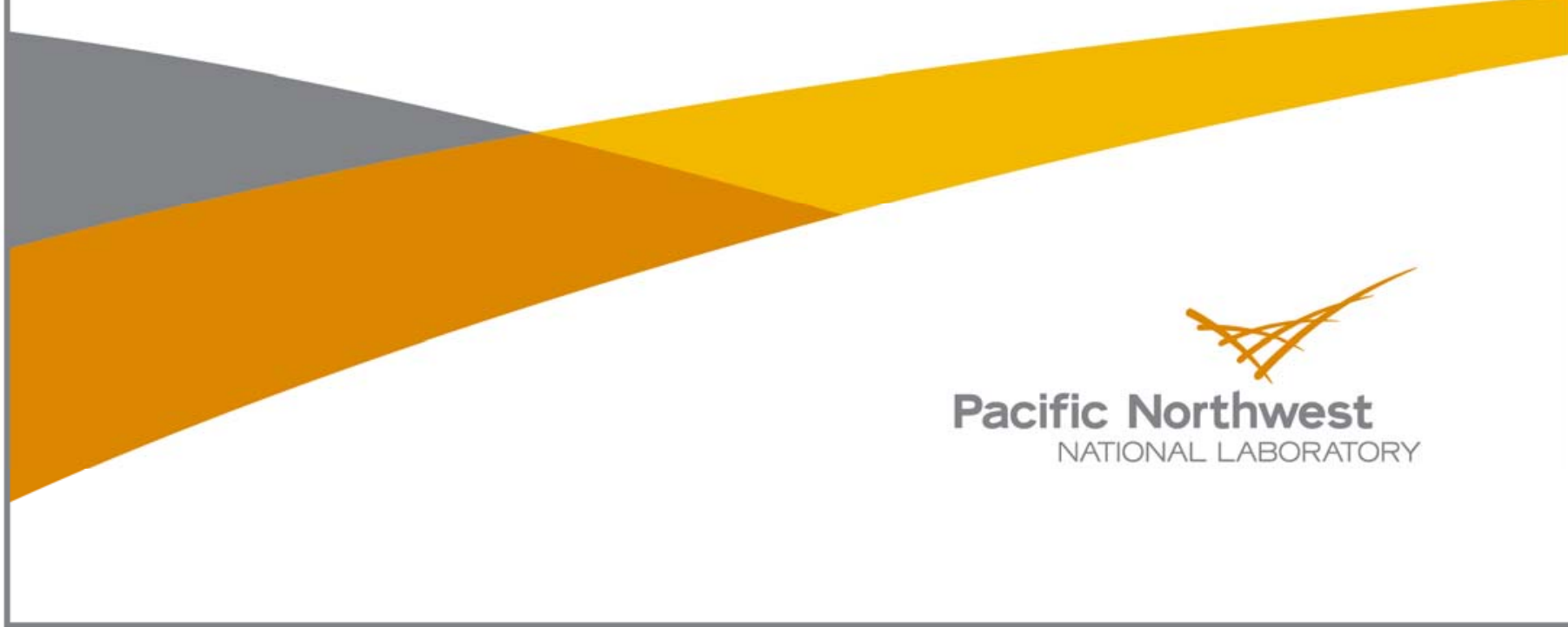


09/19/08 12:01

To: Dana Widrig

From: Michael J. Lindberg

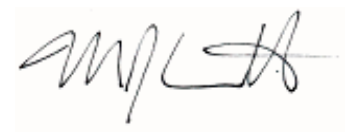

Environmental Sciences Laboratory

Energy and Environment Directorate, Pacific Northwest National Laboratory

Subject: Analytical Data Report of Water Samples Collected From BP-5 Operable Unit A Well (C5858), Sample Delivery Group ESL080006, SAF Number F08-034

This letter contains the following information for sample delivery group ESL080006

- Cover Sheet

- Narrative

- Analytical Results

- Quality Control

- Geologic Logs

- Geologic Photos

- Chain of Custodies 


\section{Introduction}

Between February 26, 2008 and March 11, 2008 groundwater samples were received from BP-5 Operable Unit A Well (C5858) for geochemical studies.

\section{Analytical Results/Methodology}

The analyses for this project were performed at the 325 building located in the 300 Area of the Hanford Site. The analyses were performed according to Pacific Northwest National Laboratory (PNNL) approved procedures and/or nationally recognized test procedures. The data sets include the sample identification numbers, analytical results, estimated quantification limits (EQL), and quality control data.

\section{Quality Control}

The preparatory and analytical quality control requirements, calibration requirements, acceptance criteria, and failure actions are defined in the on-line QA plan “Conducting Analytical Work in Support of Regulatory Programs” (CAW). This QA plan implements the Hanford Analytical Services Quality Assurance Requirements Documents (HASQARD) for PNNL.

\section{Definitions}

Dup Duplicate

RPD Relative Percent Difference

\section{Sample Receipt}

Samples were received with a chain of custody (COC) and were analyzed according to the sample identification numbers supplied by the client. All Samples were refrigerated upon receipt until prepared for analysis.

All samples were received with custody seals intact unless noted in the Case Narrative.

\section{Holding Times}

Holding time is defined as the time from sample preparation to the time of analyses. The prescribed holding times were met for all analytes unless noted in the Case Narrative.

\section{Analytical Results}

All reported analytical results meet the requirements of the CAW or client specified SOW unless noted in the case narrative. 


\section{Case Narrative Report}

Hold time:

No discrepancies noted.

Preparation Blank (PB):

No discrepancies noted.

\section{Duplicate (DUP):}

No discrepancies noted.

Laboratory control samples (LCS):

No discrepancies noted.

Post spike (PS) and post spike duplicate (PSD):

No discrepancies noted.

Matrix spike (MS) and matrix spike duplicate (MSD):

No discrepancies noted.

Other QC Criteria:

No discrepancies noted.

\section{DISCLAIMER}

This report was prepared as an account of work sponsored by an agency of the United States Government. Neither the United States Government nor any agency thereof, nor Battelle Memorial Institute, nor any of their employees, makes any warranty, express or implied, or assumes any legal liability or responsibility for the accuracy, completeness, or usefulness of any information, apparatus, product, or process disclosed, or represents that its use would not infringe privately owned rights. Reference herein to any specific commercial product, process, or service by trade name, trademark, manufacturer, or otherwise does not necessarily constitute or imply its endorsement, recommendation, or favoring by the United States Government or any agency thereof, or Battelle Memorial Institute. The views and opinions of authors expressed herein do not necessarily state or reflect those of the United States Government or any agency thereof.

\section{SAMPLES INCLUDED IN THIS REPORT}

\section{BP 5 OU, C5858 A-Well VZ}

$\begin{array}{lll}\text { HEIS No. } & \text { Laboratory ID } & \text { Matrix } \\ \text { B1T1V7 } & 0802035-01 & \text { WATER } \\ \text { B1TML2 } & 0802035-02 & \text { WATER }\end{array}$

Date Collected
2/22/08 $13: 20$
3/10/08 $14: 00$

Date Received 2/26/08 13:50

$3 / 11 / 08 \quad 11: 30$ 
The following analyses were performed on the following samples included in this report:

Anions By Ion Chromatography

Alkalinity, Titrimetic ( $\mathrm{pH} 4.5)$

Metals Water by ICPMS

Metals Water by ICPOES

$\mathrm{pH}$ of Waters By Electrode

Specific Conductance

Tc_U Water by ICPMS

\section{SAMPLES ANALYZED IN THIS REPORT}

\section{HEIS No.}

B1T1V7

B1TML2

$\begin{array}{ll}\text { Laboratory ID } & \text { Matrix } \\ \text { 0802035-01 } & \text { WATER } \\ \text { 0802035-02 } & \text { WATER }\end{array}$

\author{
Date Collected \\ 2/22/08 13:20 \\ 3/10/08 14:00
}

Date Received

2/26/08 13:50

$3 / 11 / 08 \quad 11: 30$ 


\section{Wet Chemistry}

Alkalinity as CaCO3 (ug/mL) by Standard Methods 2320B

Lab ID HEIS No.

Results

EQL

Analyzed

Batch

0802035-01

B1T1V7

1.32E2

N/A

4/02/08

8D02001

0802035-02

B1TML2

$1.25 \mathrm{E} 2$

N/A

$4 / 02 / 08$

8D02001 


\section{Wet Chemistry}

Specific Conductance (EC) (mS/cm) by EPA 120.1

Lab ID HEIS No. Results

0802035-01 B1T1V7

8.47E-1 5.00E-3

Analyzed

Batch

0802035-02

B1TML2

9.71E-1 5.00E-3

$4 / 02 / 08$

8D01001

$4 / 02 / 08$

8D01001 


\section{Wet Chemistry}

pH (pH Units) by AGG-pH-001

HEIS No.

Results

EQL

Analyzed

Batch

0802035-01

B1T1V7

7.77E0

N/A

$4 / 02 / 08$

8D01001

0802035-02

B1TML2

7.99E0

N/A

$4 / 02 / 08$

8D01001 


\section{Anions by Ion Chromatography}

\begin{tabular}{|c|c|c|c|c|c|c|c|}
\hline CAS \# & Analyte & Results & Units & EQL & Analyzed & Batch & Method \\
\hline HEIS No. & B1T1V7 & \multicolumn{3}{|c|}{ Lab ID: $\quad$ 0802035-01 } & & & \\
\hline 16984-48-8 & Fluoride & $1.18 \mathrm{E} 0$ & $\mathrm{ug} / \mathrm{mL}$ & $2.00 \mathrm{E}-1$ & 4/03/08 & 8D02005 & AGG-IC-001 \\
\hline 16887-00-6 & Chloride & $2.25 \mathrm{E} 1$ & $\mathrm{ug} / \mathrm{mL}$ & $5.00 \mathrm{E}-1$ & 4/03/08 & 8D02005 & AGG-IC-001 \\
\hline $14797-65-0$ & Nitrite & 2.02E1 & $\mathrm{ug} / \mathrm{mL}$ & $1.00 \mathrm{E} 0$ & 4/03/08 & 8D02005 & AGG-IC-001 \\
\hline 24959-67-9 & Bromide & $<1.00 \mathrm{E} 0$ & $\mathrm{ug} / \mathrm{mL}$ & $1.00 \mathrm{E} 0$ & 4/03/08 & 8D02005 & AGG-IC-001 \\
\hline 14797-55-8 & Nitrate & 8.04E1 & $\mathrm{ug} / \mathrm{mL}$ & $1.00 \mathrm{E} 0$ & 4/03/08 & 8D02005 & AGG-IC-001 \\
\hline 14808-79-8 & Sulfate & $1.42 \mathrm{E} 2$ & $\mathrm{ug} / \mathrm{mL}$ & $1.50 \mathrm{E} 0$ & 4/03/08 & 8D02005 & AGG-IC-001 \\
\hline $14265-44-2$ & Phosphate & $<1.50 \mathrm{E} 0$ & $\mathrm{ug} / \mathrm{mL}$ & $1.50 \mathrm{E} 0$ & $4 / 03 / 08$ & 8D02005 & AGG-IC-001 \\
\hline HEIS No. & B1TML2 & \multicolumn{3}{|c|}{ Lab ID: $\quad 0802035-02$} & & & \\
\hline 16984-48-8 & Fluoride & $3.01 \mathrm{E}-1$ & $\mathrm{ug} / \mathrm{mL}$ & $2.00 \mathrm{E}-1$ & 4/03/08 & 8D02005 & AGG-IC-001 \\
\hline $16887-00-6$ & Chloride & $2.00 \mathrm{E} 1$ & $\mathrm{ug} / \mathrm{mL}$ & $5.00 \mathrm{E}-1$ & $4 / 03 / 08$ & 8D02005 & AGG-IC-001 \\
\hline $14797-65-0$ & Nitrite & $<1.00 \mathrm{E} 0$ & $\mathrm{ug} / \mathrm{mL}$ & $1.00 \mathrm{E} 0$ & $4 / 03 / 08$ & 8D02005 & AGG-IC-001 \\
\hline 24959-67-9 & Bromide & $<1.00 \mathrm{E} 0$ & $\mathrm{ug} / \mathrm{mL}$ & $1.00 \mathrm{E} 0$ & 4/03/08 & 8D02005 & AGG-IC-001 \\
\hline 14797-55-8 & Nitrate & 2.06E2 & $\mathrm{ug} / \mathrm{mL}$ & $1.00 \mathrm{E} 1$ & $4 / 04 / 08$ & 8D02005 & AGG-IC-001 \\
\hline 14808-79-8 & Sulfate & $1.26 \mathrm{E} 2$ & $\mathrm{ug} / \mathrm{mL}$ & $1.50 \mathrm{E} 0$ & $4 / 03 / 08$ & 8D02005 & AGG-IC-001 \\
\hline 14265-44-2 & Phosphate & $<1.50 \mathrm{E} 0$ & $\mathrm{ug} / \mathrm{mL}$ & $1.50 \mathrm{E} 0$ & 4/03/08 & 8D02005 & AGG-IC-001 \\
\hline
\end{tabular}


Total Metals by PNNL-AGG-ICP-AES

\begin{tabular}{|c|c|c|c|c|c|c|c|}
\hline CAS \# & Analyte & Results & Units & EQL & Analyzed & Batch & Method \\
\hline HEIS No. & B1T1V7 & \multicolumn{2}{|c|}{ Lab ID: } & 0802035-01 & & & \\
\hline $7429-90-5$ & Aluminum & $<5.22 \mathrm{E} 1$ & ug/L & 5.22E1 & $5 / 01 / 08$ & 8D11003 & PNNL-AGG-ICP-AES \\
\hline $7440-39-3$ & Barium & 6.87E1 & ug/L & $1.95 \mathrm{E} 1$ & $5 / 01 / 08$ & 8D11003 & PNNL-AGG-ICP-AES \\
\hline $7440-70-2$ & Calcium & $5.55 \mathrm{E} 4$ & ug/L & 8.66E1 & $5 / 01 / 08$ & 8D11003 & PNNL-AGG-ICP-AES \\
\hline $7440-48-4$ & Cobalt & $<2.31 \mathrm{E} 1$ & ug/L & 2.31E1 & $5 / 01 / 08$ & 8D11003 & PNNL-AGG-ICP-AES \\
\hline $7440-47-3$ & Chromium & $<1.07 \mathrm{E} 1$ & ug/L & $1.07 \mathrm{E} 1$ & $5 / 01 / 08$ & 8D11003 & PNNL-AGG-ICP-AES \\
\hline $7440-50-8$ & Copper & $<1.27 \mathrm{E} 1$ & ug/L & $1.27 \mathrm{E} 1$ & $5 / 01 / 08$ & 8D11003 & PNNL-AGG-ICP-AES \\
\hline 7439-89-6 & Iron & $<2.96 \mathrm{E} 1$ & ug/L & 2.96E1 & $5 / 01 / 08$ & 8D11003 & PNNL-AGG-ICP-AES \\
\hline $7440-09-7$ & Potassium & $1.18 \mathrm{E} 4$ & ug/L & $1.52 \mathrm{E} 3$ & $5 / 01 / 08$ & 8D11003 & PNNL-AGG-ICP-AES \\
\hline 7439-95-4 & Magnesium & $2.05 \mathrm{E} 4$ & ug/L & $1.50 \mathrm{E} 1$ & $5 / 01 / 08$ & 8D11003 & PNNL-AGG-ICP-AES \\
\hline 7439-96-5 & Manganese & $6.19 \mathrm{E} 2$ & ug/L & 7.62E0 & $5 / 01 / 08$ & 8D11003 & PNNL-AGG-ICP-AES \\
\hline $7440-02-0$ & Nickel & $<2.84 \mathrm{E} 1$ & ug/L & $2.84 \mathrm{E} 1$ & $5 / 01 / 08$ & 8D11003 & PNNL-AGG-ICP-AES \\
\hline $7782-49-2$ & Selenium & $<7.70 \mathrm{E} 2$ & ug/L & 7.70E2 & $5 / 01 / 08$ & 8D11003 & PNNL-AGG-ICP-AES \\
\hline $7440-62-2$ & Vanadium & $<1.48 \mathrm{E} 2$ & ug/L & $1.48 \mathrm{E} 2$ & $5 / 01 / 08$ & 8D11003 & PNNL-AGG-ICP-AES \\
\hline $7440-66-6$ & Zinc & $<5.06 \mathrm{E} 1$ & ug/L & $5.06 \mathrm{E} 1$ & $5 / 01 / 08$ & 8D11003 & PNNL-AGG-ICP-AES \\
\hline $7440-23-5$ & Sodium & $6.44 \mathrm{E} 4$ & ug/L & $6.48 \mathrm{E} 2$ & $5 / 01 / 08$ & 8D11003 & PNNL-AGG-ICP-AES \\
\hline 7704-34-9 & Sulfur & $5.10 \mathrm{E} 4$ & ug/L & $5.52 \mathrm{E} 2$ & $5 / 01 / 08$ & 8D11003 & PNNL-AGG-ICP-AES \\
\hline HEIS No. & B1TML2 & \multicolumn{2}{|c|}{ Lab ID: } & 0802035-02 & & & \\
\hline 7429-90-5 & Aluminum & $<5.22 \mathrm{E} 1$ & ug/L & 5.22E1 & $5 / 01 / 08$ & 8D11003 & PNNL-AGG-ICP-AES \\
\hline $7440-39-3$ & Barium & $1.01 \mathrm{E} 2$ & ug/L & $1.95 \mathrm{E} 1$ & $5 / 01 / 08$ & 8D11003 & PNNL-AGG-ICP-AES \\
\hline $7440-70-2$ & Calcium & $9.41 \mathrm{E} 4$ & ug/L & 8.66E1 & $5 / 01 / 08$ & 8D11003 & PNNL-AGG-ICP-AES \\
\hline $7440-48-4$ & Cobalt & $<2.31 \mathrm{E} 1$ & ug/L & $2.31 \mathrm{E} 1$ & $5 / 01 / 08$ & 8D11003 & PNNL-AGG-ICP-AES \\
\hline $7440-47-3$ & Chromium & $<1.07 \mathrm{E} 1$ & ug/L & $1.07 \mathrm{E} 1$ & $5 / 01 / 08$ & 8D11003 & PNNL-AGG-ICP-AES \\
\hline $7440-50-8$ & Copper & $<1.27 \mathrm{E} 1$ & ug/L & $1.27 \mathrm{E} 1$ & $5 / 01 / 08$ & 8D11003 & PNNL-AGG-ICP-AES \\
\hline 7439-89-6 & Iron & $<2.96 \mathrm{E} 1$ & ug/L & $2.96 \mathrm{E} 1$ & $5 / 01 / 08$ & 8D11003 & PNNL-AGG-ICP-AES \\
\hline 7440-09-7 & Potassium & $9.20 \mathrm{E} 3$ & ug/L & $1.52 \mathrm{E} 3$ & $5 / 01 / 08$ & 8D11003 & PNNL-AGG-ICP-AES \\
\hline 7439-95-4 & Magnesium & $2.80 \mathrm{E} 4$ & ug/L & $1.50 \mathrm{E} 1$ & $5 / 01 / 08$ & 8D11003 & PNNL-AGG-ICP-AES \\
\hline $7439-96-5$ & Manganese & 3.70E1 & ug/L & 7.62E0 & $5 / 01 / 08$ & 8D11003 & PNNL-AGG-ICP-AES \\
\hline 7440-02-0 & Nickel & $<2.84 \mathrm{E} 1$ & ug/L & $2.84 \mathrm{E} 1$ & $5 / 01 / 08$ & 8D11003 & PNNL-AGG-ICP-AES \\
\hline $7782-49-2$ & Selenium & $<7.70 \mathrm{E} 2$ & $\mathrm{ug} / \mathrm{L}$ & $7.70 \mathrm{E} 2$ & $5 / 01 / 08$ & 8D11003 & PNNL-AGG-ICP-AES \\
\hline $7440-62-2$ & Vanadium & $<1.48 \mathrm{E} 2$ & $\mathrm{ug} / \mathrm{L}$ & $1.48 \mathrm{E} 2$ & $5 / 01 / 08$ & 8D11003 & PNNL-AGG-ICP-AES \\
\hline $7440-66-6$ & Zinc & $1.94 \mathrm{E} 2$ & $\mathrm{ug} / \mathrm{L}$ & $5.06 \mathrm{E} 1$ & $5 / 01 / 08$ & 8D11003 & PNNL-AGG-ICP-AES \\
\hline $7440-23-5$ & Sodium & $2.91 \mathrm{E} 4$ & ug/L & $6.48 \mathrm{E} 2$ & $5 / 01 / 08$ & 8D11003 & PNNL-AGG-ICP-AES \\
\hline 7704-34-9 & Sulfur & $4.49 \mathrm{E} 4$ & $\mathrm{ug} / \mathrm{L}$ & $5.52 \mathrm{E} 2$ & $5 / 01 / 08$ & 8D11003 & PNNL-AGG-ICP-AES \\
\hline
\end{tabular}


Radionuclides By ICP-MS

\begin{tabular}{|c|c|c|c|c|c|c|c|}
\hline CAS \# & Analyte & Results & Units & EQL & Analyzed & Batch & Method \\
\hline HEIS No. & B1T1V7 & \multicolumn{2}{|c|}{ Lab ID: } & 0802035-01 & & & \\
\hline \multirow[t]{2}{*}{$14133-76-7$} & Technetium-99 & 3.31E-2 & ug/L & 2.35E-2 & 4/16/08 & 8D16003 & PNNL-AGG-415 \\
\hline & Uranium 238 & 2.35E2 & ug/L & $1.31 \mathrm{E}-1$ & 4/16/08 & 8D16003 & PNNL-AGG-415 \\
\hline HEIS No. & B1TML2 & \multicolumn{2}{|c|}{ Lab ID: } & 0802035-02 & & & \\
\hline \multirow[t]{2}{*}{ 14133-76-7 } & Technetium-99 & 1.33E0 & ug/L & 4.70E-1 & 4/16/08 & 8D16003 & PNNL-AGG-415 \\
\hline & Uranium 238 & 4.11E3 & ug/L & 2.62E0 & 4/16/08 & 8D16003 & PNNL-AGG-415 \\
\hline
\end{tabular}


RCRA Metals By PNNL-AGG-415

\begin{tabular}{|c|c|c|c|c|c|c|c|}
\hline CAS \# & Analyte & Results & Units & EQL & Analyzed & Batch & Method \\
\hline HEIS No. & B1T1V7 & \multicolumn{2}{|c|}{ Lab ID: } & 0802035-01 & & & \\
\hline $14378-38-2$ & Silver & $1.78 \mathrm{E}-1$ & ug/L & $7.40 \mathrm{E}-2$ & $4 / 24 / 08$ & 8D21002 & PNNL-AGG-415 \\
\hline 14336-64-2 & Cadmium & $6.15 \mathrm{E}-1$ & ug/L & $1.65 \mathrm{E}-1$ & $4 / 24 / 08$ & 8D21002 & PNNL-AGG-415 \\
\hline $14265-72-6$ & Antimony & $1.81 \mathrm{E} 0$ & ug/L & $1.20 \mathrm{E}-1$ & $4 / 24 / 08$ & 8D21002 & PNNL-AGG-415 \\
\hline HEIS No. & B1TML2 & \multicolumn{2}{|c|}{ Lab ID: } & 0802035-02 & & & \\
\hline 14378-38-2 & Silver & $<7.40 \mathrm{E}-2$ & ug/L & $7.40 \mathrm{E}-2$ & $4 / 24 / 08$ & 8D21002 & PNNL-AGG-415 \\
\hline $14336-64-2$ & Cadmium & 2.09E-1 & ug/L & $1.65 \mathrm{E}-1$ & $4 / 24 / 08$ & 8D21002 & PNNL-AGG-415 \\
\hline $14265-72-6$ & Antimony & $2.30 \mathrm{E}-1$ & ug/L & $1.20 \mathrm{E}-1$ & $4 / 24 / 08$ & 8D21002 & PNNL-AGG-415 \\
\hline
\end{tabular}




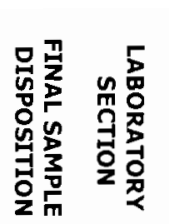

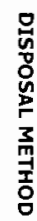

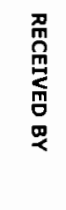

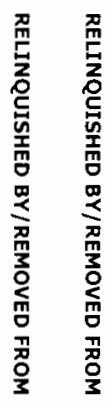

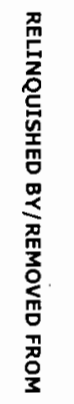

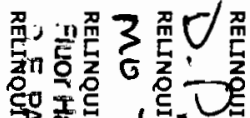

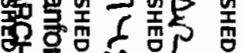

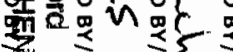

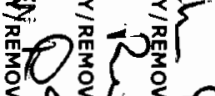

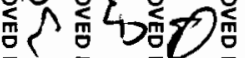

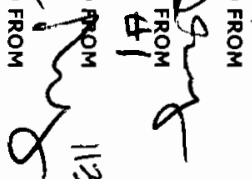

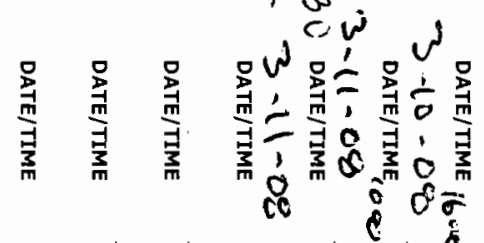

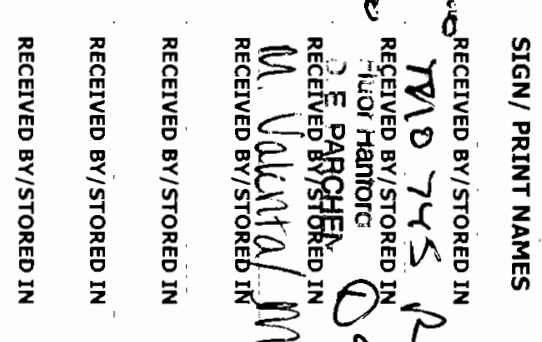

$\sum \xi_{0}^{\infty}$

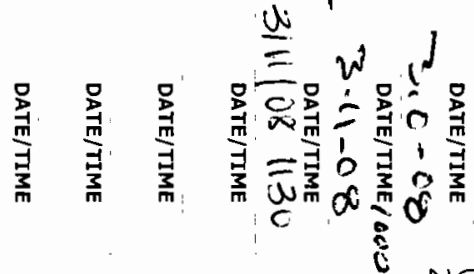

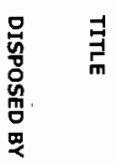

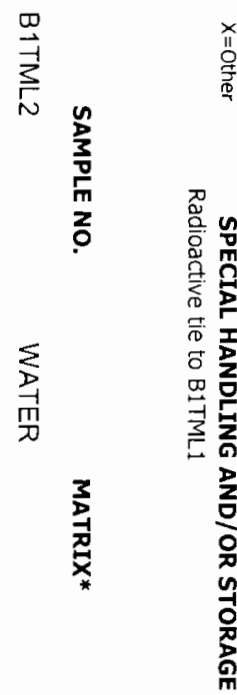

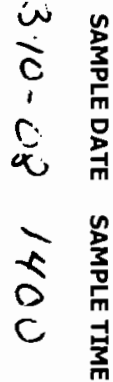

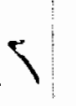

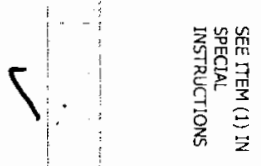

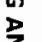

음

ํํㅁ

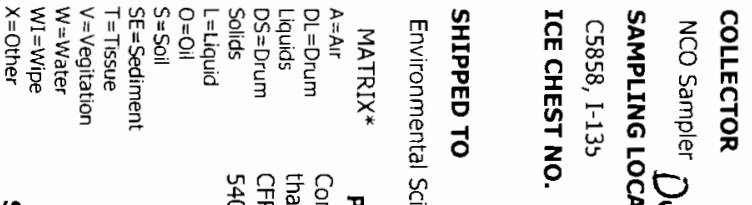
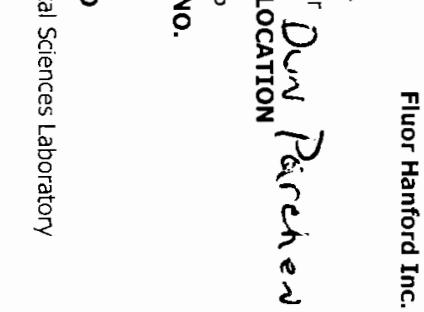

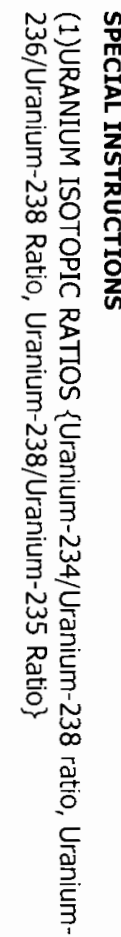

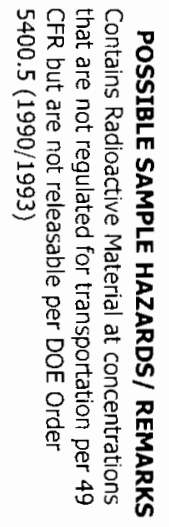

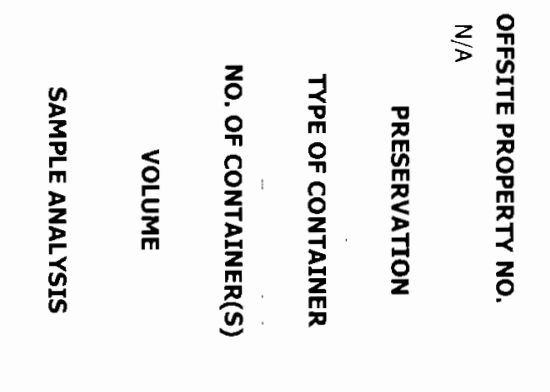

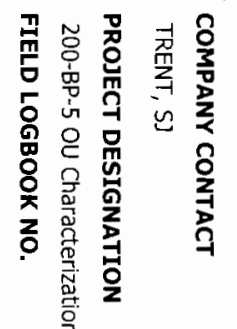

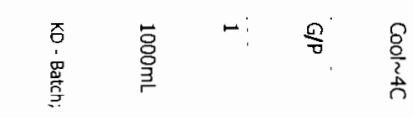

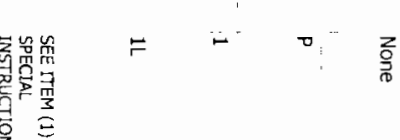

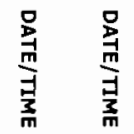




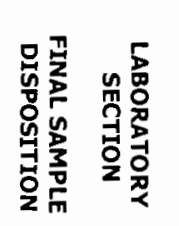

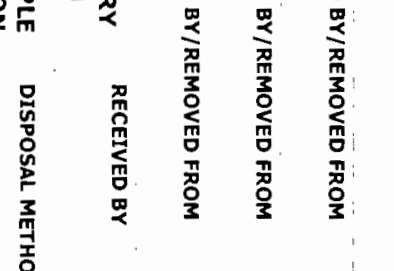

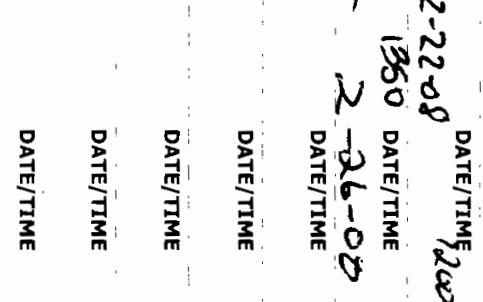

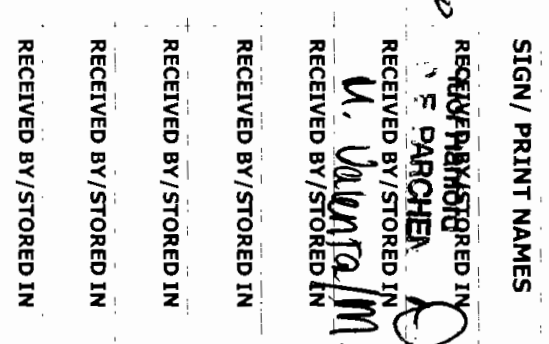

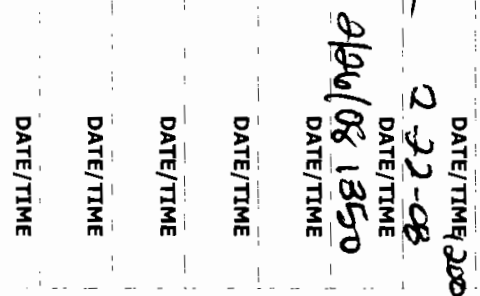

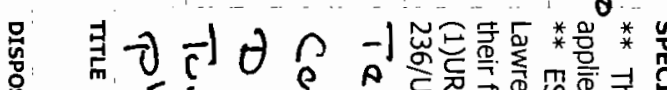
$\sum 0$ ही

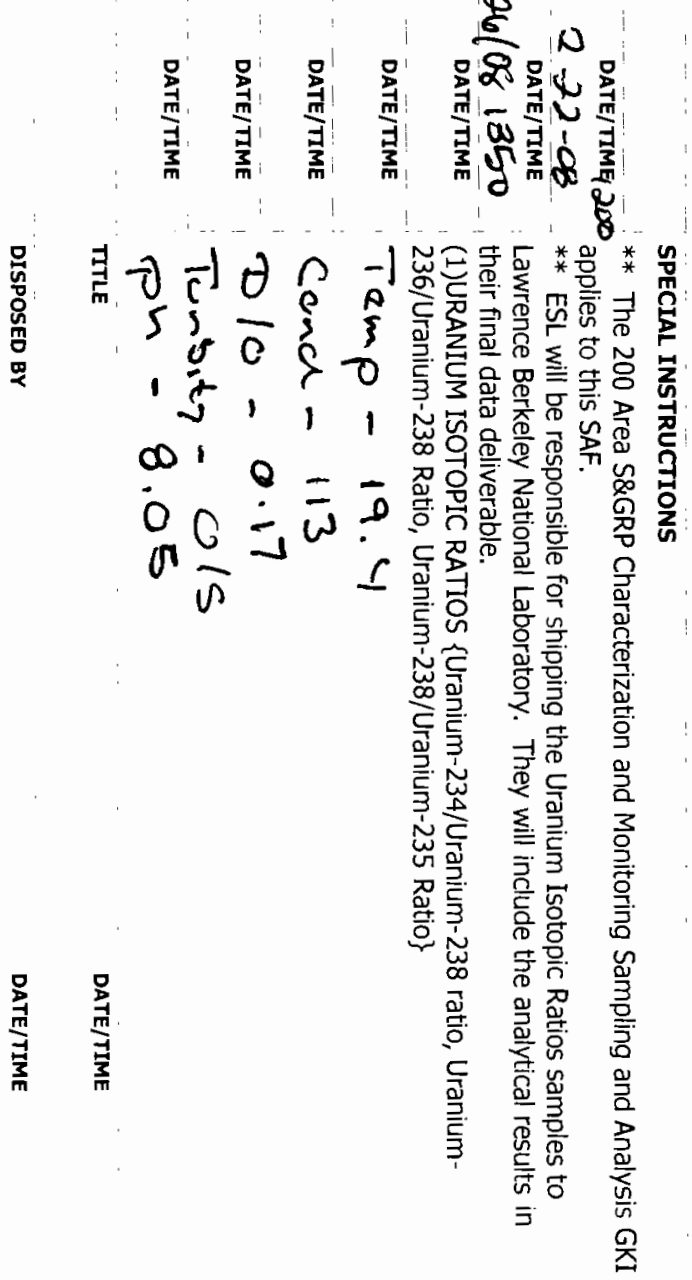

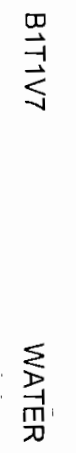

弪

$\frac{N}{\frac{N}{N}}$
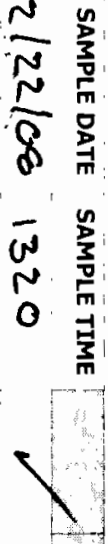

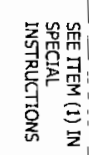

ํㅡㅇ

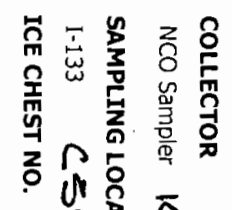

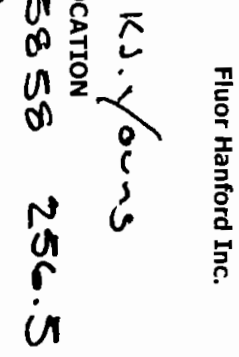

㖇完产

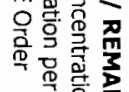

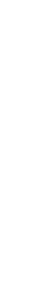

